\title{
LIGHTLIKE HYPERSURFACES IN SEMI-RIEMANNIAN MANIFOLD WITH SEMI-SYMMETRIC NON-METRIC CONNECTION
}

\author{
EROL YAŞAR, A. CEYLAN ÇÖKEN and AHMET YÜCESAN
}

\begin{abstract}
In this paper, we study lightlike hypersurfaces of a semi-Riemannian manifold admitting a semisymmetric non-metric connection. We give the equations of Gauss and Codazzi. Then, we obtain conditions under which the Ricci tensor of a lightlike hypersurface is symmetric given that the ambient space is equipped with a semi-symmetric non-metric connection.
\end{abstract}

\section{Introduction}

Ageshe and Chafle [1] introduced the idea of a semi-symmetric non-metric connection on a Riemannian manifold. Prasad and Verma [11] got the necessary and sufficient condition in order that the Weyl projective curvature tensor of a semi-symmetric non-metric connection is equal to the projective curvature of the Riemannian connection. In addition, they obtained a necessary and sufficient condition for the Ricci tensor of the semi-symmetric non-metric connection to be symmetric. De and Kamilya [4] gave basic properties of a hypersurface of a Riemannian manifold with semi-symmetric non-metric connection.

In this paper we study lightlike hypersurface of a semi-Riemannian manifold admitting the semi-symmetric non-metric connection. It is well known that lightlike hypersurfaces are of metrics with vanishing determinants and this degeneracy of these metrics leads to several difficulties: Firstly, the contravariant metric cannot immediately be defined, so the connection cannot be specified uniquely in the normal way. Secondly, the normal is a lightlike vector lying in the tangent plane, which makes it necessary to look for some other vector to rig the hypersurface, and makes it impossible to normalise the normal in the usual way. Since these objects are considered, to study the general theory of lightlike hypersurfaces is very important topic. Several papers have been written on lightlike hypersurfaces in recent years [2], [3], [7], [9].

Received June 13, 2006; in revised form November 24, 2006. 
In the present paper, we have proved that on lightlike hypersurfaces the connection induced from a semi-symmetric non-metric connection is semi-symmetric non-metric, and also on the screen distribution the connection induced from that connection is semi-symmetric non-metric. For a lightlike hypersurface and screen distribution we define the induced geometrical objects with respect to a semi-symmetric non-metric connection such as second fundamental form, shape operator, etc., ... Then we give equations of Gauss and Codazzi. Finally, we obtain the conditions under which the Ricci tensor of a lightlike hypersurface with respect to a semi-symmetric non-metric connection is symmetric. Also, we show that the Ricci tensor of a lightlike hypersurface is not parallel with respect to a semi-symmetric non-metric connection.

In our preceding work [14], we study totally umbilical lightlike hypersurfaces of a semi-Riemannian manifold admitting a semi-symmetric metric connection. We find that the induced connection on a totally umbilical lightlike hypersurface from a semi-symmetric metric connection is a semi-symmetric but not metric. However we show that the induced connection on the screen distribution is metric. Then, we obtain some results on Ricci tensor of a totally umbilical lightlike hypersurface with respect to a semi-symmetric connection.

\section{Preliminaries}

Let $M$ be a hypersurface of a $(n+1)$-dimensional, $n>1$, semi-Riemannian manifold $\widetilde{M}$ with semi-Riemannian metric $\widetilde{g}$ of index $1 \leq v \leq n$. We consider

$$
T_{x} M^{\perp}=\left\{Y_{x} \in T_{x} \tilde{M} \mid \tilde{g}_{x}\left(Y_{x}, X_{x}\right)=0, \forall X_{x} \in T_{x} M\right\}
$$

for any $x \in M$. Then we say that $M$ is a lightlike (null, degenerate) hypersurface of $\widetilde{M}$ or equivalently, the immersion

$$
i: M \rightarrow \tilde{M}
$$

of $M$ in $\widetilde{M}$ is lightlike (null, degenerate) if $T_{x} M \cap T_{x} M^{\perp} \neq\{0\}$ at any $x \in$ $M$. Henceforth we identify $i(M)$ with $M$ and we denote the differential $d i$, immersing a vector field $X$ on $M$ to a vector field $\phi X$ on $\widetilde{M}$, by $\phi$. Thus the induced metric tensor $g=\widetilde{g}_{\left.\right|_{M}}$ is defined by

$$
g(X, Y)=\tilde{g}(\phi X, \phi Y), \quad \forall X, Y \in \Gamma(T M) .
$$

An orthogonal complementary vector bundle of $T M^{\perp}$ in $T M$ is non-degenerate subbundle of $T M$ called the screen distribution on $M$ and denoted $S(T M)$. We have the following splitting into orthogonal direct sum:

$$
T M=S(T M) \perp T M^{\perp} .
$$


The subbundle $S(T M)$ is non-degenerate, so is $S(T M)^{\perp}$, and the following holds:

$$
T \tilde{M}=S(T M) \perp S(T M)^{\perp},
$$

where $S(T M)^{\perp}$ is the orthogonal complementary vector bundle to $S(T M)$ in $\left.T \tilde{M}\right|_{M}$.

Let $\operatorname{tr}(T M)$ denote the complementary vector bundle of $T M^{\perp}$ in $S(T M)^{\perp}$. Then we have

$$
S(T M)^{\perp}=T M^{\perp} \oplus \operatorname{tr}(T M) .
$$

Let $\mathscr{U}$ be a coordinate neighborhood in $M$ and $\xi$ be a basis of $\Gamma\left(\left.T M^{\perp}\right|_{\mathscr{U}}\right)$. Then there exists a basis $N$ of $\left.\operatorname{tr}(T M)\right|_{\mathscr{U}}$ satisfying the following conditions:

$$
\tilde{g}(N, \xi)=1
$$

and

$$
\tilde{g}(N, N)=\tilde{g}(W, N)=0, \quad \forall W \in \Gamma\left(\left.S(T M)\right|_{\mathscr{U}}\right) .
$$

The subbundle $\operatorname{tr}(T M)$ is called a lightlike transversal vector bundle of $M$. We note that $\operatorname{tr}(T M)$ is never orthogonal to $T M$. From (1), (2) and (3) we have

$$
\left.T \tilde{M}\right|_{M}=S(T M) \perp\left(T M^{\perp} \oplus \operatorname{tr}(T M)\right)=T M \oplus \operatorname{tr}(T M)
$$

EXAMPLE 2.1 ([5]). In $R_{2}^{4}$ consider the hypersurface

$$
M=\left\{\left(x_{1}, x_{2}, x_{3}, x_{4}\right) \mid x_{4}=x_{1}+\frac{1}{2}\left(x_{2}+x_{3}\right)^{2}\right\} .
$$

It is easy to check that $M$ is a lightlike hypersurface and

$$
T M^{\perp}=\operatorname{Span}\{\xi\}
$$

where $\xi=\left(1, x_{2}+x_{3},-x_{2}-x_{3}, 1\right)$. Then take as lightlike transversal vector bundle

$$
\operatorname{tr}(T M)=\operatorname{Span}\{N\},
$$

where $N=-\frac{1}{2\left(1+\left(x_{2}+x_{3}\right)^{2}\right)}\left(1, x_{2}+x_{3}, x_{2}+x_{3},-1\right)$. It follows that the corresponding screen distribution $S(T M)$ is spanned by

$$
\left\{W_{1}=\left(-x_{2}-x_{3}, 1,0,0\right), W_{2}=\left(0,0,1, x_{2}+x_{3}\right)\right\} \text {. }
$$




\section{Semi-Symmetric Non-Metric Connection}

Let $\widetilde{M}$ be an $(n+1)$-dimensional, $n>1$, differentiable manifold of class $C^{\infty}$ and $\widetilde{\nabla}$ a linear connection in $\widetilde{M}$. If the torsion tensor $\widetilde{T}$ of $\widetilde{\nabla}$ defined by

$$
\widetilde{T}(\tilde{X}, \tilde{Y})=\widetilde{\nabla}_{\widetilde{X}} \widetilde{Y}-\widetilde{\nabla}_{\widetilde{Y}} \widetilde{X}-[\tilde{X}, \tilde{Y}], \quad \forall \widetilde{X}, \widetilde{Y} \in \Gamma(T \tilde{M})
$$

satisfies

$$
\widetilde{T}(\tilde{X}, \tilde{Y})=\tilde{\pi}(\tilde{Y}) \tilde{X}-\tilde{\pi}(\tilde{X}) \tilde{Y}
$$

for a 1 -form $\tilde{\pi}$, then the connection $\widetilde{\nabla}$ is said to be semi-symmetric ([1] and [13]).

Let a semi-Riemannian metric $\tilde{g}$ of index $v$ with $1 \leq v \leq n$ in $\tilde{M}$ be given and $\tilde{\nabla}$ satisfy

$$
\left(\widetilde{\nabla}_{\tilde{X}} \tilde{g}\right)(\tilde{Y}, \widetilde{Z})=-\tilde{\pi}(\tilde{Y}) \widetilde{g}(\tilde{X}, \widetilde{Z})-\tilde{\pi}(\widetilde{Z}) \widetilde{g}(\tilde{X}, \tilde{Y}),
$$

then such a linear connection is called a non-metric connection [1].

We now suppose that the semi-Riemannian manifold $\widetilde{M}$ admits a semisymmetric non-metric connection given by

$$
\widetilde{\nabla}_{\widetilde{X}} \widetilde{Y}=\stackrel{\odot}{\nabla}_{\widetilde{X}} \tilde{Y}+\widetilde{\pi}(\tilde{Y}) \tilde{X}
$$

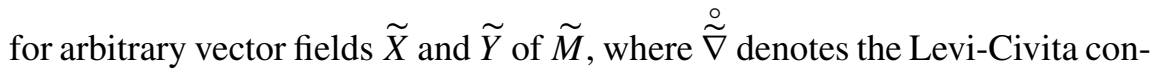
nection with respect to the semi-Riemannian metric $\widetilde{g}, \tilde{\pi}$ is a 1-form associated with the vector field $\widetilde{Q}$ on $\widetilde{M}$ by

$$
\tilde{\pi}(\tilde{X})=\widetilde{g}(\tilde{X}, \widetilde{Q})
$$

(see [1] and [6]).

By using the second form of the decomposition (4), we can write

$$
\widetilde{Q}=\phi Q+\mu N
$$

where $Q$ is a vector field and $\mu$ is a function in $M$.

We know that the Gauss formula with respect to $\stackrel{\circ}{\nabla}$ the symmetric linear connection induced on the lightlike hypersurface from $\stackrel{\stackrel{\nabla}{\nabla}}{\text { is }}$

$$
\stackrel{\circ}{\nabla}_{\phi X} \phi Y=\phi\left(\stackrel{\circ}{\nabla}_{X} Y\right)+B(X, Y) N
$$

for any $X, Y \in \Gamma(T M)$, where $B$ is the second fundamental form of $M$ [5]. Denoting by $\nabla$ the connection induced on the lightlike hypersurface from $\tilde{\nabla}$, 
we have

$$
\tilde{\nabla}_{\phi X} \phi Y=\phi\left(\nabla_{X} Y\right)+m(X, Y) N
$$

where $m$ is a tensor of type $(0,2)$ of the lightlike hypersurface of $M$ and we call (8) the Gauss formula with respect to the induced connection $\nabla$.

In view of (5), we find

$$
\widetilde{\nabla}_{\phi X} \phi Y=\stackrel{\circ}{\widetilde{\nabla}}_{\phi X} \phi Y+\tilde{\pi}(\phi Y) \phi X
$$

and therefore, using (7) and (8), we can also write

$$
\begin{aligned}
\phi\left(\nabla_{X} Y\right)+m(X, Y) N=\phi & \left(\stackrel{\circ}{\nabla}_{X} Y\right)+B(X, Y) N \\
& +\tilde{\pi}(\phi Y) \phi X .
\end{aligned}
$$

Substituting (6) into (9), we obtain

$$
\phi\left(\nabla_{X} Y\right)+m(X, Y) N=\phi\left(\stackrel{\circ}{\nabla}_{X} Y+\pi(Y) X\right)+B(X, Y) N,
$$

from which

$$
\nabla_{X} Y=\stackrel{\circ}{\nabla}_{X} Y+\pi(Y) X
$$

where $\pi(X)=\tilde{\pi}(\phi X)$ and

$$
m(X, Y)=B(X, Y)
$$

Taking account of (10) and the connection induced on lightlike hypersurface from Levi-Civita connection is not metric, we obtain

$$
\begin{aligned}
\left(\nabla_{X} g\right)(Y, Z)=m(X, Y) \eta(Z)+ & m(X, Z) \eta(Y) \\
& -\pi(Y) g(X, Z)-\pi(Z) g(X, Y)
\end{aligned}
$$

where

$$
\eta(Z)=\tilde{g}(Z, N)
$$

for any $X, Y, Z \in \Gamma(T M)$ and $N \in \Gamma(\operatorname{tr}(T M))$. We also get from (10)

$$
T(X, Y)=\pi(Y) X-\pi(X) Y .
$$

Thus, from (12) and (13), we have

Proposition 3.1. The connection induced on a lightlike hypersurface of a semi-Riemannian manifold with a semi-symmetric non-metric connection is also a semi-symmetric non-metric connection. 
The Weingarten formula with respect to the Levi-Civita connection $\stackrel{\stackrel{\nabla}{\nabla}}{\text { is }}$

$$
\stackrel{\circ}{\nabla}_{\phi X} N=-\phi\left(\stackrel{\circ}{A}_{N} X\right)+\tau(X) N
$$

for any vector field $X$ of $M$, where $\stackrel{\circ}{A}_{N}$ is the shape operator of $M$ and $\tau$ is a 1-form [5].

Next, if $P$ denotes the projection morphism of $T M$ on $S(T M)$ with respect to the decomposition (1) and using (5), we get

$$
\widetilde{\nabla}_{\phi X} N=\stackrel{0}{\nabla}_{\phi X} N+\lambda \phi X,
$$

because of

$$
\tilde{\pi}(N)=\widetilde{g}(\widetilde{Q}, N)=\widetilde{g}(P \phi Q+\lambda \xi+\mu N, N)=\lambda g(\xi, N)=\lambda .
$$

Thus using (14), we find

$$
\widetilde{\nabla}_{\phi X} N=-\phi\left(\left(\stackrel{\circ}{A}_{N}-\lambda I\right) X\right)+\tau(X) N,
$$

where $I$ is the unit tensor. Denoting $A_{N}$ by $A_{N}=\left(\AA_{N}-\lambda I\right)$ and from (15), we have

$$
\widetilde{\nabla}_{\phi X} N=-\phi\left(A_{N} X\right)+\tau(X) N
$$

for any vector field $X$ of $M$. We call (16) the Weingarten formula with respect to the semi-symmetric non-metric connection.

We know that the geometry of a lightlike hypersurface depends on the chosen screen distribution and the local fundamental form with respect to the symmetric connection of $M$ on $\mathscr{U}$ is independent of the choice of screen distribution [5]. Then from (11), we have

Proposition 3.2. Let $S(T M)$ and $S(T M)^{\prime}$ be two screen distributions on $M$ and $m$ and $m^{\prime}$ be the second fundamental forms with respect to $\operatorname{tr}(T M)$ and $\operatorname{tr}(T M)^{\prime}$, respectively. Then $m=m^{\prime}$ on $\mathcal{U}$, that is the local second fundamental form of $M$ on $\mathcal{U}$, is independent of the choice of screen distribution.

COROLlaRY 3.3. The second fundamental form with respect to a semisymmetric non-metric connection of a lightlike hypersurface is degenerate.

Proof. Taking into account that $\widetilde{\nabla}$ is a semi-symmetric non-metric connection, from Proposition 3.2 it follows that

$$
m(X, \xi)=0, \quad \forall X \in \Gamma(T M)
$$

which proves the assertion. 
Now, we give geometrical objects for screen distribution. Since $P$ denotes the projection morphism of $T M$ on $S(T M)$ with respect to the decomposition (1), we have

$$
\stackrel{\circ}{\nabla}_{\phi X} P \phi Y=\phi\left(\stackrel{\circ}{\nabla}_{X} P Y\right)+C(X, P Y) \xi
$$

and

$$
\stackrel{\circ}{\nabla}_{\phi X} \xi=-\phi\left(\stackrel{\circ}{A}_{\xi} X\right)-\tau(X) \xi
$$

for any $X, Y \in \Gamma(T M)$ and $\xi \in \Gamma\left(T M^{\perp}\right)$, where $\phi\left(\stackrel{\circ}{\nabla^{\nabla}} X Y\right)$ and $\phi\left(\stackrel{\circ}{A_{\xi}^{*}} X\right)$ belong to $\Gamma(S(T M))$ and $C$ is 1 -form on $M$ such that $C$ is defined by

$$
C(X, P Y)=g\left(\stackrel{\circ}{A}_{N} X, P Y\right)
$$

[5]. Analogous to the equation (17), we have

$$
\nabla_{\phi X} P \phi Y=\phi\left(\stackrel{*}{\nabla}_{X} P Y\right)+D(X, P Y) \xi,
$$

where $\phi\left(\vec{\nabla}_{X} P Y\right)$ belong to $\Gamma(S(T M))$ and $D$ is 1 -form on $M$.

From (10), we get

$$
\nabla_{\phi X} P \phi Y=\stackrel{\circ}{\nabla}_{\phi X} P \phi Y+\pi(P \phi Y) \phi X .
$$

Thus, using (17) and (20), we have

$$
\phi\left(\stackrel{*}{\nabla}_{X} P Y\right)+D(X, P Y) \xi=\phi\left(\stackrel{\circ}{\nabla_{X}} P Y\right)+C(X, P Y) \xi+\pi(P \phi Y) \phi X
$$

from which

$$
\stackrel{*}{\nabla}_{X} P Y=\stackrel{\circ}{\nabla} \text { 要 } P Y+\pi(P Y) X
$$

and

$$
D(X, P Y)=C(X, P Y)+\pi(P Y) \widetilde{g}(X, N)
$$

for any $X \in \Gamma(T M)$. 
By using (21), we find

$$
\begin{aligned}
\stackrel{*}{\nabla}_{X}(g(P Y, P Z))= & \left(\stackrel{*}{\nabla}_{X} g\right)(P Y, P Z)+\stackrel{\circ}{\nabla}_{X}(g(P Y, P Z))-\left(\stackrel{*}{\nabla}_{X} g\right)(P Y, P Z) \\
& +\pi(P Y) g(P X, P Z)+\pi(P Z) g(P X, P Y)
\end{aligned}
$$

from this, we obtain

$$
\left(\stackrel{*}{\nabla}_{X} g\right)(P Y, P Z)=-\pi(P Y) g(P X, P Z)-\pi(P Z) g(P X, P Y) .
$$

We also have from (21)

$$
\stackrel{*}{T}(P X, P Y)=\pi(P Y) P X-\pi(P X) P Y .
$$

Then, in view of (23) and (24), we have

Proposition 3.4. The connection $\stackrel{*}{\nabla}$ induced on a screen distribution of lightlike hypersurface is a semi-symmetric non-metric connection.

\section{Equations of Gauss and Codazzi}

We denote by

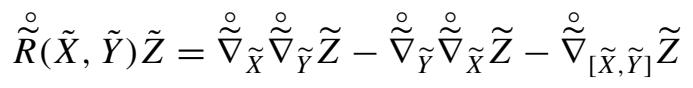

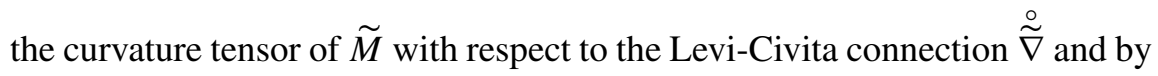

$$
\stackrel{\circ}{R}(X, Y) Z=\stackrel{\circ}{\nabla}_{X} \stackrel{\circ}{\nabla}_{Y} Z-\stackrel{\circ}{\nabla}_{Y} \stackrel{\circ}{\nabla}_{X} Z-\stackrel{\circ}{\nabla}_{[X, Y]} Z
$$

that of $M$ with respect to induced connection $\stackrel{\circ}{\nabla}$. Then the Gauss-Codazzi equations of lightlike hypersurface are given by

$$
\begin{aligned}
& \tilde{g}(\stackrel{\circ}{\widetilde{R}}(\phi X, \phi Y) \phi Z, \phi P W)=g(\stackrel{\circ}{R}(X, Y) Z, P W) \\
& +B(X, Z) C(Y, P W)-B(Y, Z) C(X, P W), \\
& \text { (26) } \tilde{g}(\stackrel{\circ}{R}(\phi X, \phi Y) \phi Z, \xi)=\left(\stackrel{\circ}{\nabla}_{X} B\right)(Y, Z)-\left(\stackrel{\circ}{\nabla}_{Y} B\right)(X, Z) \\
& +B(Y, Z) \tau(X)-B(X, Z) \tau(Y),
\end{aligned}
$$

and

$$
\widetilde{g}(\stackrel{\circ}{R}(\phi X, \phi Y) \phi Z, N)=\widetilde{g}(\stackrel{\circ}{R}(\phi X, \phi Y) \phi Z, N)
$$


for any $X, Y, Z, W \in \Gamma(T M), \xi \in \Gamma\left(T M^{\perp}\right), N \in \Gamma(\operatorname{tr}(T M))$ [5].

Now, we shall find the equations of Gauss and Codazzi of lightlike hypersurface with a semi-symmetric non-metric connection. The curvature tensor of the semi-symmetric non-metric connection $\widetilde{\nabla}$ of $\widetilde{M}$ is, by definition,

$$
\widetilde{R}(\widetilde{X}, \widetilde{Y}) \widetilde{Z}=\widetilde{\nabla}_{\widetilde{X}} \widetilde{\nabla}_{\widetilde{Y}} \widetilde{Z}-\widetilde{\nabla}_{\widetilde{Y}} \widetilde{\nabla}_{\widetilde{X}} \widetilde{Z}-\widetilde{\nabla}_{[\tilde{X}, \tilde{Y}]} \widetilde{Z}
$$

Putting $\widetilde{X}=\phi X, \tilde{Y}=\phi Y, \widetilde{Z}=\phi Z$, we get

$$
\widetilde{R}(\phi X, \phi Y) \phi Z=\widetilde{\nabla}_{\phi X} \widetilde{\nabla}_{\phi Y} \phi Z-\widetilde{\nabla}_{\phi Y} \widetilde{\nabla}_{\phi X} \phi Z-\widetilde{\nabla}_{\phi[X, Y]} \phi Z .
$$

Thus, using (8) and (16), we find (28)

$$
\begin{aligned}
\widetilde{R}(\phi X, \phi Y) \phi Z=\phi & (R(X, Y) Z)+m(X, Z) A_{N} Y-m(Y, Z) A_{N} X \\
& +\left\{m(\pi(Y) X-\pi(X) Y, Z)+\left(\nabla_{X} m\right)(Y, Z)\right. \\
& \left.-\left(\nabla_{Y} m\right)(X, Z)+m(Y, Z) \tau(X)-m(X, Z) \tau(Y)\right\} N
\end{aligned}
$$

where $R(X, Y) Z=\nabla_{X} \nabla_{Y} Z-\nabla_{Y} \nabla_{X} Z-\nabla_{[X, Y]} Z$ is the curvature tensor of a lightlike hypersurface with a semi-symmetric non-metric connection $\nabla$.

Then, we obtain from (16), (19), (22) and (28)

$$
\begin{aligned}
& \widetilde{g}(\widetilde{R}(\phi X, \phi Y) \phi Z, \phi P W) \\
&=g(R(X, Y) Z, P W)+\lambda m(Y, Z) g(P X, P W) \\
&-\lambda m(X, Z) g(P Y, P W)+m(X, Z) D(Y, P W) \\
&-m(Y, Z) D(X, P W)+m(Y, Z) \pi(P W) \xi(X) \\
&-m(X, Z) \pi(P W) \xi(Y),
\end{aligned}
$$

$$
\begin{array}{r}
\tilde{g}(\tilde{R}(\phi X, \phi Y) \phi Z, \xi)=m(\pi(Y) X-\pi(X) Y, Z)+\left(\nabla_{X} m\right)(Y, Z) \\
-\left(\nabla_{Y} m\right)(X, Z)+m(Y, Z) \tau(X)-m(X, Z) \tau(Y),
\end{array}
$$

and

$$
\begin{aligned}
\widetilde{g}(\widetilde{R}(\phi X, \phi Y) \phi Z, N)=\widetilde{g}(R(\phi X, \phi Y) \phi Z, N) & +\lambda m(Y, Z) \eta(X) \\
& -\lambda m(X, Z) \eta(Y) .
\end{aligned}
$$

We call equations (29)-(31) the Gauss-Codazzi equations of the lightlike hypersurface with a semi-symmetric non-metric connection. 


\section{The Ricci Tensor of Lightlike Hypersurface with Semi-Symmetric Non-Metric Connection}

Let $M$ be a lightlike hypersurface of a $(n+1)$-dimensional, $n>1$, semiRiemannian manifold $\widetilde{M}$ admitting a semi-symmetric non-metric connection. Analogous to the definition of the Ricci tensor of $M$ with respect to the symmetric connection, we define the Ricci tensor of $M$ with respect to a semisymmetric non-metric connection by

$$
\operatorname{Ric}(X, Y)=\operatorname{trace}\{Z \longrightarrow R(X, Z) Y\},
$$

for any $X, Y, Z \in \Gamma(T M)$. Then, the Ricci tensor of $M$ with respect to a semi-symmetric non-metric connection is given by

$$
\operatorname{Ric}(X, Y)=\sum_{i=1}^{n-1} \varepsilon_{i} g\left(R\left(X, W_{i}\right) Y, W_{i}\right)+\widetilde{g}(R(X, \xi) Y, N)
$$

where $\left\{W_{1}, \ldots, W_{n-1}\right\}$ are the orthonormal basis of screen distribution.

Thus, by using (10), (28) and (33) we obtain

$$
\operatorname{Ric}(X, Y)-\operatorname{Ric}(Y, X)=2 d \tau(X, Y)+(n-1) d \pi(X, Y)
$$

for any $X, Y \in \Gamma(T M)$.

From (34), we have

Proposition 5.1. Let $M$ be a lightlike hypersurface of a semi-Riemannian manifold $\widetilde{M}$ admitting a semi-symmetric non-metric connection. Then Ricci tensor of a lightlike hypersurface with respect to a semi-symmetric non-metric connection is symmetric if and only if the 1-forms $\tau$ and $\pi$ are closed.

We assume that the 1 -form $\pi$ is closed. Then we can define the sectional curvature for a section in $\widetilde{M}$ with respect to the semi-symmetric non-metric connection (see [1]).

Now, suppose that the semi-symmetric non-metric connection $\widetilde{\nabla}$ is of constant sectional curvature, then $\widetilde{R}(\widetilde{X}, \widetilde{Y}) \widetilde{Z}$ should be of the form

$$
\widetilde{R}(\tilde{X}, \tilde{Y}) \widetilde{Z}=c\{\widetilde{g}(\tilde{X}, \widetilde{Z}) \tilde{Y}-\widetilde{g}(\tilde{Y}, \widetilde{Z}) \tilde{X}\}
$$

for any $\tilde{X}, \widetilde{Y}, \widetilde{Z} \in \Gamma(T \widetilde{M})$, where $c$ is a certain scalar. Thus $\widetilde{M}$ is a semiRiemannian manifold of constant curvature $c$ with respect to semi-symmetric non-metric connection and denote it by $\widetilde{M}(c)$.

Proposition 5.2. Let $M$ be a lightlike hypersurface of an $(n+1)$-dimensional semi-Riemannian space form $\widetilde{M}(c)$ with a semi-symmetric non-metric 
connection. Then we get

$$
\operatorname{Ric}(X, Y)=(n-1) c g(X, Y)-H m(X, Y)+\sum_{i=1}^{n-1} \varepsilon_{i} m\left(W_{i}, Y\right) D\left(X, W_{i}\right),
$$

for any $X, Y, Z \in \Gamma(T M)$, where $H$ is the mean curvature of lightlike hypersurface with respect to semi-symmetric non-metric connection given by

$$
H=\sum_{i=1}^{n-1} \varepsilon_{i} g\left(A_{N} W_{i}, W_{i}\right)
$$

Proof. Substituting (28) into (33) and taking into account (35), we obtain (36).

COROLlary 5.3. Let $M$ be a lightlike hypersurface of an $(n+1)$-dimensional semi-Riemannian space form $\tilde{M}(c)$ with a semi-symmetric non-metric connection. Then the Ricci tensor of $M$ is symmetric if and only if the shape operator with respect to a semi-symmetric non-metric connection of $M$ is symmetric with respect to the second fundamental form of $M$.

Proposition 5.4. Let $M$ be a lightlike hypersurface of an $(n+1)$-dimensional, $n>1$, semi-Riemannian space form $\tilde{M}(c)$ with a semi-symmetric non-metric connection. Then Ricci tensor of $M$ is not parallel with respect to semi-symmetric non-metric connection $\nabla$.

Proof. If we take the derivative of Ricci tensor of $M$ with respect to a semi-symmetric non-metric connection, we find

$$
\left(\nabla_{Z} \operatorname{Ric}\right)(X, Y)=\nabla_{Z} \operatorname{Ric}(X, Y)-\operatorname{Ric}\left(\nabla_{Z} X, Z\right)-\operatorname{Ric}\left(X, \nabla_{Z} Y\right) .
$$

On the other hand, using equations (36) and (12), we have

$$
\begin{aligned}
& \left(\nabla_{Z} \operatorname{Ric}\right)(X, Y) \\
& =(n-1) c\left\{\left(\nabla_{Z} g\right)(X, Y)-\left(\nabla_{Z} m\right)(X, Y) H-m(X, Y) Z(H)\right. \\
& \quad+\sum_{i=1}^{n-1} \varepsilon_{i}\left\{\left(\nabla_{Z} m\right)\left(W_{i}, Y\right)+m\left(\nabla_{Z} W_{i}, Y\right)\right\} D\left(X, W_{i}\right) \\
& \quad+\sum_{i=1}^{n-1} \varepsilon_{i} m\left(W_{i}, Y\right)\left\{\left(\nabla_{Z} D\right)\left(X, W_{i}\right)+D\left(X, \nabla_{Z} W_{i}\right)\right\} .
\end{aligned}
$$

Since $\nabla$ is a semi-symmetric non-metric connection on $M$, the term $\left(\nabla_{Z} g\right)(X, Y)$ in the right hand side of equation (37) is not vanishing for any $X, Y, Z \in \Gamma(T M)$. So the proof is completed. 


\section{REFERENCES}

1. Ageshe, N. S., and Chafle, M. R., A semi-symmetric non-metric connection on a Riemannian manifold, Indian J. Pure Appl. Math. 23 (1992), No. 6, 399-409.

2. Bejancu, A., Null Hypersurfaces in semi-Euclidean space, Saitama Math. J. 14 (1996), 25-40.

3. Bejancu, A., and Duggal, K. L., Lightlike submanifolds of semi-Riemannian manifolds, Acta Appl. Math. 38 (1995) No. 2, 197-215.

4. De, U. C., and Kamilya, D., Hypersurfaces of a Riemannian manifold with semi-symmetric non-metric connection, J. Indian Inst. Sci. 75 (1995), No. 6, 707-710.

5. Duggal, K. L., and Bejancu, A., Lightlike submanifolds of semi-Riemannian manifolds and applications, Kluwer Academic Pub., The Netherlands (1996).

6. Duggal, K. L., and Sharma, R., Semi-symmetric metric connections in a semi-Riemannian manifold, Indian J. Pure Appl. Math. 17 (1986), 1276-1283.

7. Güneş, R., Şahin, B., and Kılıç, E., On lightlike hypersurfaces of a semi-Riemannian space form, Turkish J. Math. 27 (2003), No. 2, 283-297.

8. Hayden, H. A., Subspaces of a space with torsion, Proc. London Math. Soc. II Ser. 34 (1932), 27-50.

9. Katsuno, K., Null hypersurfaces in Lorentzian manifolds I, Math. Proc. Cambridge Philos. Soc. 88 (1980), 175-182.

10. O'Neill, B., Semi-Riemannian Geometry with Applications to Relativity, Academic Press, London (1983).

11. Prasad, B., and Verma, R. K., On a type of semi-symmetric non-metric connection on a Riemannian manifold, Bull. Calcutta Math. Soc. 96 (2004), No. 6, 483-488.

12. Sengupta, J., De, U. C., and Binh, T. Q., On a type of semi-symmetric non-metric connection on a Riemannian manifold, Indian J. Pure Appl. Math. 31 (2000), No. 12, 1659-1670.

13. Yano, K., On semi-symmetric metric connection, Rev. Roumaine Math. Pures Appl. 15 (1970), 1579-1586.

14. Yaşar, E., Çöken, A. C., and Yücesan, A., Totally umbilical lightlike hypersurfaces in semiRiemannian manifold with semi-symmetric metric connection, Int. J. Pure and Appl. Math. 23 (2005), No. 3, 379-391.

ULA VOCATIONAL HIGH SCHOOL

MUĞLA UNIVERSITY

ULA, MUĞLA

TURKEY

E-mail: yerol@mu.edu.tr

DEPARTMENT OF MATHEMATICS

SÜLEYMAN DEMIREL UNIVERSITY

32260 ISPARTA

TURKEY

E-mail: yucesan@fef.sdu.edu.tr
DEPARTMENT OF MATHEMATICS

SÜLEYMAN DEMIREL UNIVERSITY 32260 ISPARTA

TURKEY

E-mail: ceylan@fef.sdu.edu.tr 